\title{
BMJ Open Effect of weekly teriparatide injections on osteoporotic fracture healing: protocol for a double-blind, randomised controlled trial
}

\author{
Junxiong Zhu, ${ }^{1}$ Chenggui Zhang, ${ }^{1}$ Jialin Jia, ${ }^{1}$ Wanqiong Yuan, ${ }^{1,2}$ Min Zhang, ${ }^{1}$ \\ Huijie Leng, ${ }^{1,2}$ Chunli Song (i) ${ }^{1,2}$
}

To cite: Zhu J, Zhang C, Jia J, et al. Effect of weekly teriparatide injections on osteoporotic fracture healing: protocol for a double-blind, randomised controlled trial. BMJ Open 2021;11:e043137. doi:10.1136/ bmjopen-2020-043137

\section{- Prepublication history and} additional supplemental material for this paper are available online. To view these files, please visit the journal online (http://dx.doi.org/10.1136/ bmjopen-2020-043137).

Received 25 July 2020 Revised 06 February 2021 Accepted 17 March 2021

\section{Check for updates}

(c) Author(s) (or their employer(s)) 2021. Re-use permitted under CC BY-NC. No commercial re-use. See rights and permissions. Published by BMJ.

${ }^{1}$ Department of Orthopedics, Peking University Third Hospital, Beijing, China

${ }^{2}$ Beijing Key Laboratory of Spinal Diseases, Beijing, China

Correspondence to Professor Chunli Song; schl@bjmu.edu.cn

\section{ABSTRACT}

Introduction Both animal studies and clinical trials have shown that daily parathyroid hormone administration promotes bone fracture healing. We previously found that weekly injections of the recombinant human parathyroid hormone teriparatide at a dosage of $20 \mu \mathrm{g} / \mathrm{kg}$ promoted tibial fracture healing to the same extent as daily injections of teriparatide at a dosage of $10 \mu \mathrm{g} / \mathrm{kg}$ in a rodent model. However, the effect of weekly teriparatide administration on human fracture healing is unreported. This protocol describes a randomised controlled clinical trial designed to evaluate whether weekly administration of teriparatide accelerates fracture repair in humans.

Methods and analysis This single-centre, doubleblind, randomised controlled trial will be conducted in Peking University Third Hospital. Eligible patients with Colles' fracture incurred within 48 hours will be randomly divided into two groups ( $\mathrm{n}=40$ per group) that will receive 14 weekly subcutaneous injections of either saline or teriparatide ( $40 \mu \mathrm{g} / \mathrm{week}$ ). The primary outcome will be the time taken to achieve radiographic healing, as assessed using the modified radiographic union scale for tibial fractures. The secondary outcomes will be functional assessments, including the self-administered PatientRated Wrist Evaluation questionnaire, grip strength and rate of fracture non-union.

Ethics and dissemination Ethical approval has been obtained from the Peking University Third Hospital Medical Science Research Ethics Committee (M2020207). The findings will be disseminated in peer-reviewed publications.

Trial registration number NCT04473989: protocol version: 1

\section{INTRODUCTION}

Osteoporosis affects more than 200 million women and causes 8.9 million fractures per year worldwide. ${ }^{1}$ Osteoporotic fractures are very challenging to treat and are associated with high mortality and disability rates and medical costs. The long healing time of osteoporotic fractures leads to prolonged pain and immobilisation, which greatly decrease quality of life. Accelerating the fracture
Strengths and limitations of this study

- This is the first trial to study the effect of weekly teriparatide injections on the healing of osteoporotic fractures.

- This clinical trial is based on the results of animal experiments, which could increase the probability of positive outcomes.

- The double-blind study design will increase the validity of the results.

- A limitation of this trial is that it does not have a multicentre design.

healing not only helps to reduce the pain, but also improves the quality of life, which is important for reducing the consumption of resources from a socioeconomic aspect.

The current adjuvant therapies used to accelerate fracture healing are very limited. Since Heckman et al first reported in 1994 that low-intensity pulsed ultrasound (LIPUS) accelerates human bone healing, LIPUS has been studied for the treatment and prevention of osteoporotic fractures. However, its effect is still controversial. ${ }^{3}$ A large, multicentre, blind, randomised controlled clinical trial showed that LIPUS did not accelerate the radiographic fracture healing and the functional recovery. ${ }^{4}$ Another adjuvant therapy option is the administration of bone morphogenetic protein (BMP)-2, which has osteogenic effects and has a therapeutic effect on open tibiofibular fractures. Both preclinical and clinical studies have shown that BMP-2 promotes fracture healing; however, BMP-2 is not as effective in humans as in animals, ${ }^{56}$ and the topical application of BMP causes marked adverse effects, such as local swelling, pain, increased risk of wound infection and even an increased risk of cancer. ${ }^{7-9}$ Clinical studies funded by pharmaceutical companies clearly do not report the adverse 
effects of BMP when applied in spinal fusion. ${ }^{7}$ In addition to BMP, the use of recombinant human platelet-derived growth factor-BB $/ \beta$-tricalcium phosphate was approved in 2015 by the Food and Drug Administration (FDA) as an alternative to autografting during ankle and hindfoot fusion surgery. ${ }^{10}$ The use of adjuvant therapy to promote fracture healing is based on two considerations: one is to accelerate the recovery process of the fracture, and the other is to reduce the incidence of fracture-related complications, such as non-union. ${ }^{11}$

Parathyroid hormone (PTH) is the first antiosteoporosis drug with anabolic effects that has been approved by the FDA. ${ }^{12}$ Many animal experiments have shown that teriparatide promotes callus formation and increases the mechanical strength of fracture healing ${ }^{13-15}$ in a dose-related manner. ${ }^{16}{ }^{17}$ However, few clinical trials have evaluated whether PTH promotes fracture healing in humans. Aspenberg et $a l^{18}$ completed a prospective, double-blind, randomised controlled trial that evaluated the effects of teriparatide on the healing of distal radial fractures in 102 postmenopausal women who received either a placebo, $20 \mu \mathrm{g} /$ day of teriparatide or $40 \mu \mathrm{g} /$ day of teriparatide. The fracture healing time was significantly shorter in the $20 \mu \mathrm{g} /$ day group than the placebo group, suggesting that teriparatide accelerates fracture healing. ${ }^{18}$ This team also shows teriparatide improves early callus formation in distal radial fractures despite the analysis is outside the protocol. ${ }^{19}$ Several other studies have also shown that PTH has a certain promoting effect on fracture healing. ${ }^{20-22}$ To the best of our knowledge, no clinical studies have shown that drugs other than BMP and PTH are effective in promoting fracture healing.

Although PTH has an excellent osteogenic effect, it is expensive and requires daily subcutaneous administration. Poor patient compliance negatively impacts the therapeutic effect of PTH. ${ }^{23}$ Therefore, in recent years, the effectiveness of weekly subcutaneous injections of PTH has been assessed in the treatment of osteoporosis. Two double-blind, randomised controlled trials evaluated the effects of the weekly administration of a low dose of PTH (28.2 $\mu \mathrm{g}$ /week) and a high dose of PTH (56.5 $\mu \mathrm{g} /$ week); both drug administration strategies significantly increase the bone density of the vertebral body and reduce the risk of vertebral fracture. ${ }^{24-26}$ In addition, a multicentre, prospective randomised controlled trial showed that weekly injections of teriparatide $(56.5 \mu \mathrm{g})$ enhance intervertebral fusion. ${ }^{27}$ These studies suggest that a weekly teriparatide administration strategy promotes bone formation. In addition to studying the effects of weekly teriparatide administration on bone mineral density and fracture risk, studies have shown that daily and weekly administration strategies have different effects on bone turnover markers. ${ }^{2428} 29$ The daily administration strategy increases bone resorption markers, while weekly administration reduces bone resorption markers, and a single injection of teriparatide affects bone turnover for more than 1 week. ${ }^{29}$ Our previous animal experiments showed that weekly injections of $20 \mu \mathrm{g} / \mathrm{kg}$ of teriparatide promote bone fracture healing to the same extent as daily injections of $10 \mu \mathrm{g} / \mathrm{kg}$ of teriparatide. ${ }^{30}$ However, the effect of weekly teriparatide administration on fracture healing is still unclear, and has not been evaluated in a clinical study. This study will be the first clinical study to examine the effect of weekly teriparatide administration on fracture healing.

Despite clinicians' expectation that PTH can promote fracture healing given the results of animal studies and limited human studies, the trial results may be negatively influenced by different regimes (dose, frequency), cases or types of fractures. ${ }^{31}$ For example, Johansson has shown that $20 \mu \mathrm{g} /$ day PTH 1-34 may not improve healing in proximal humerus fractures, ${ }^{31}$ while it can accelerate the healing time of distal radial fractures. ${ }^{18}$ Based on the promising results of distal radial fractures, here we also choose Colles' fracture as the fracture type. Currently, 20 $\mu \mathrm{g} /$ day is the main treatment dose for osteoporosis and thus, it becomes the most popular dose for fracture study. However, the dose for fracture may be different from the one for osteoporosis since they have different biological mechanisms. Here we choose $40 \mu \mathrm{g}$ as the dose for weekly usage based on some clues. According to the statistical result in our previous animal fracture study, increasing the frequency at low doses can yield great benefits for fracture healing, while increasing the frequency at high doses may not be as obviously beneficial as that at low doses. This implies that once-weekly injections of a higher dose of PTH might be feasible for promoting bone fracture healing. ${ }^{30}$ Ota $e t a \vec{l}^{32}$ also demonstrated that administering teriparatide at higher doses and/or higher frequencies raises fracture callus volume in rodent model. In an osseous union enhancement study conducted by Ebata $e t$ $a l$, they found that high dose, $56.5 \mu \mathrm{g} /$ week, is effective in the healing of human lumbar interbody fusion. ${ }^{27}$

\section{METHODS AND ANALYSIS}

\section{Study design and setting}

This double-blind, single-centre, randomised controlled trial will be conducted in Peking University Third Hospital. Patient enrolment will start on 1 June 2021.

\section{Patient and public involvement}

Patients or the public will not be involved in the design, or conduct, or reporting, or dissemination plans of our research.

\section{Recruitment strategy}

Patients will be recruited from those referred to the orthopaedic department with a fresh distal radius fracture considered suitable for conservative treatment, including closed reduction and immobilisation. Eligible patients will be invited to participate and asked to provide written informed consent (see online supplemental file 1) before any study procedure occurs. Figure 1 shows the flow chart of this trial. 


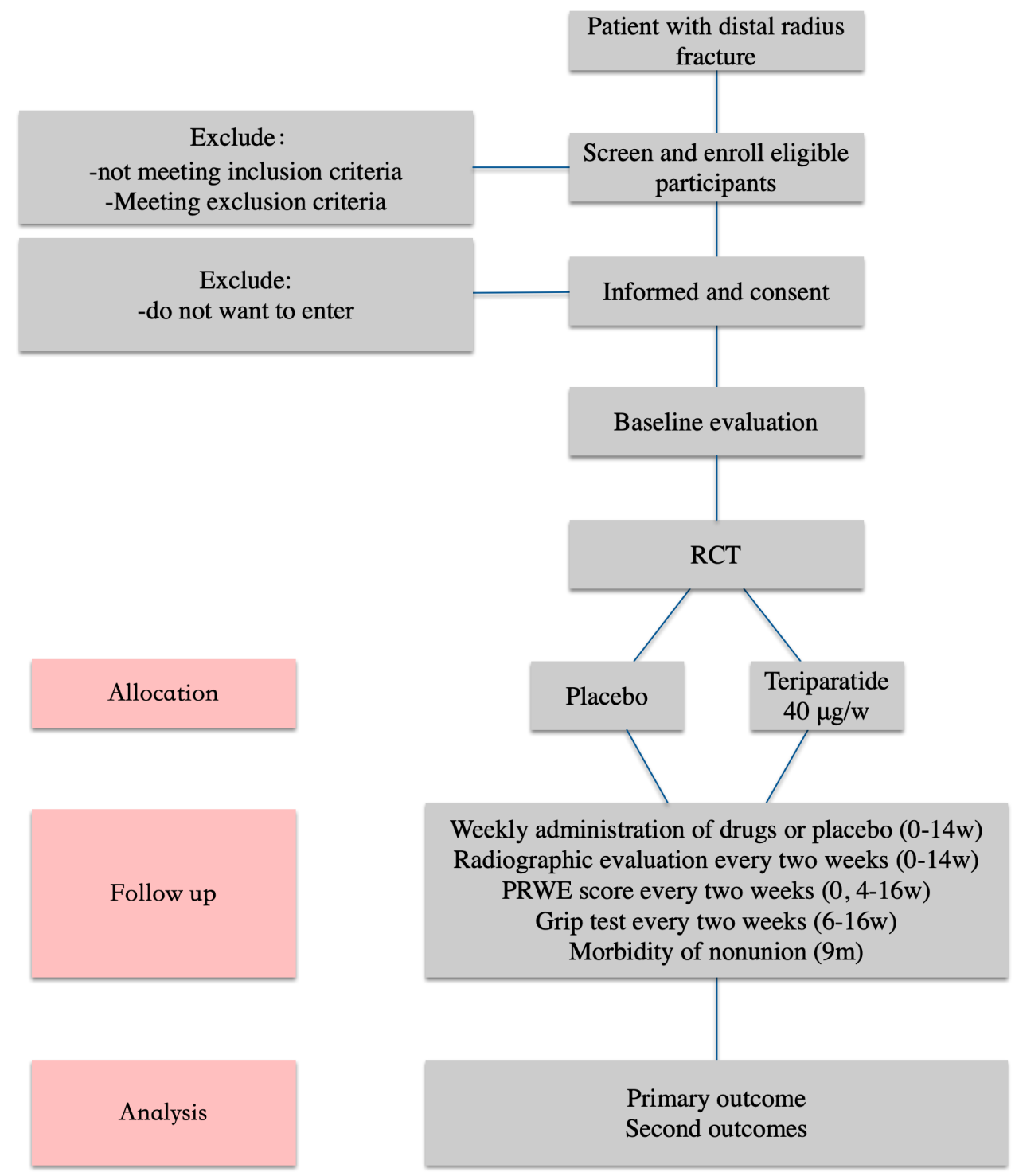

Figure 1 Flow chart of the study. PRWE, Patient-Rated Wrist Evaluation; RCT, randomised controlled trial.

\section{Patients}

Inclusion criteria

1. Postmenopausal women aged $45-75$ years (at least 2 years after menopause)

2. Primary osteoporosis.

3. Colles' fracture incurred within 48 hours.

4. Conservative fracture treatment (closed reduction and immobilisation).

5. Single fracture.

6. Provision of informed consent for trial participation.

\section{Exclusion criteria}

1. Other physical diseases, including diabetes, severe hypertension, autoimmune diseases, heart, liver and kidney diseases, malignant tumours, mental illnesses and other diseases that doctors believe may affect the healing process.

2. In addition to primary osteoporosis, any disease affecting bone metabolism or treatment response, including serum PTH $>65 \mathrm{pg} / \mathrm{mL}$, 25-hydroxyvitamin D
$<20 \mathrm{ng} / \mathrm{mL}$, alkaline phosphatase $>135 \mathrm{U} / \mathrm{L}$, history of bone tumour, Paget's disease or history of radiotherapy.

3. A history of trauma or surgery at the fracture site that may affect the function of the wrist or forearm.

4. Allergy to PTH or any excipients.

5. Currently receiving anti-osteoporosis treatment or receiving other anti-osteoporosis treatment during the trial.

6. Contraindications to the administration of teriparatide, including hyperparathyroidism, severe renal insufficiency and hypercalcaemia.

Withdrawal criteria

1. Intolerable adverse effects.

2. The patient wishes to withdraw before the end of the trial.

3. The patient is not suitable for further trial inclusion due to other accidents.

4. The patient cannot adhere to the follow-up schedule. 


\section{Baseline assessment}

Subjects will be assessed immediately after confirmation that they have met the initial eligibility criteria and provided informed consent. The baseline assessment will include the patient's age, height, weight, time from injury to the initiation of PTH treatment, Patient-Rated Wrist Evaluation (PRWE) score, energy of the trauma that caused the fracture (low energy or high energy), whether the fracture was displaced, and whether the fracture was combined with an ulnar styloid fracture, comminution or impaction.

\section{Intervention}

Patients will be randomly assigned to either the placebo group or the teriparatide group. Patients will receive 14-week teriparatide ( $40 \mu \mathrm{g} /$ week) or placebo injection. In addition to the teriparatide/placebo intervention, all patients will receive basic supplements, including 1000 $\mathrm{mg}$ /day of elemental calcium and $800 \mathrm{IU} /$ day of vitamin $\mathrm{D}$, from the screening phase to the 14-week assessment. Patients will also be provided with standardised advice on fracture protection, swelling control, skin care and everyday activities, and given an exercise programme to follow at home. The exercise programme will be progressive and consist of active range of motion exercises for the shoulder, elbow, wrist and hand, soft tissue stretches, isometric stabilising wrist exercises, and gentle forearm/ wrist/hand strengthening exercises (including grip strengthening exercises) from week $3 .^{33}$ In an effort to maximise compliance, participants will be provided with a booklet illustrating these exercises and must show this booklet to a specific nurse at every hospital visit.

Participants must visit the hospital once a week to receive a subcutaneous injection of either saline (as a placebo) or teriparatide from an assigned nurse. Fracture healing will be evaluated radiographically every 2 weeks for 14 weeks.

\section{Study outcomes}

Primary outcome

This study was designed to investigate the effect of weekly teriparatide administration on fracture healing. The primary outcome measure is the time taken to achieve radiographic healing (using the modified radiographic union scale for tibial fractures (RUST) scoring system). ${ }^{34}$ The median time taken to achieve radiographic healing will be statistically analysed to see whether teriparatide decreases the fracture healing time compared with the placebo. The most widely used fracture screening or fracture healing evaluation method is plain radiography. Clinicians in the orthopaedics are very familiar with fracture assessment via plain radiography. In addition, this method is widely used in hospitals of all levels, the radiation volume is relatively low compared with CT, and the price for repeated evaluations is affordable. The use of plain radiography to evaluate fracture healing achieves a high level of consistency among surgeons and imaging specialists. Studies have shown that the modified RUST system is better than the RUST system in evaluating metaphyseal injuries, and is reliable for evaluating the healing of metaphyseal fractures. ${ }^{34}$ The modified RUST system will be used to score each cortex on anteroposterior and lateral radiographs as 1 (no callus), 2 (callus present), 3 (bridging callus present) or 4 (fracture has remodelled and is no longer visible). The modified RUST score is the sum of these scores and therefore ranges from 4 to 16 . A modified RUST score of 13 indicates fracture union.

Radiographs will be assessed by three orthopaedists who have undertaken standardised training that involves the review of similar cases and a discussion of the criteria for defining the modified RUST scores. Score assessments will be performed in batches after all patients have completed all visits. The assessors will be blinded to the patients' group assignments, but not to the sequential order of the visits. The final result will be the average healing time based on the modified RUST scores assigned by the three assessors.

\section{Secondary outcomes}

Secondary outcomes will mainly focus on functional assessments, including the PRWE score and grip strength. The PRWE is designed to reduce the influence of subjective factors. ${ }^{35}$ The self-administered PRWE is a 15 -item questionnaire that rates wrist-related pain and disability during six specific functional tasks; the patient rates his or her ability to perform the usual level of function in the domains of self-care, work, household work and recreation. The PRWE score ranges from 0 (no disability) to 100 (severest disability). A negative per cent change from baseline in the PRWE score reflects an improvement in function. The PRWE score will be evaluated at the time of enrolment and at 4, 6, 8, 10, 12, 14 and 16 weeks. Grip strength will be assessed by a dynamometer. To adjust for hand dominance in grip strength, if the non-dominant hand was injured, the percentage will be multiplied by 1.07; if the dominant hand was injured, the percentage will be multiplied by $0.93 .{ }^{18}$ The grip strength will be tested at $6,8,10,12,14$ and 16 weeks.

According to the criteria of the American Academy of Orthopaedic Surgeons, fracture non-union is diagnosed if there is no healing at least 9 months after fracture occurrence or during continuous dynamic observation for 3 months. The incidence of fracture non-union will be assessed by telephone follow-up at 9 months after fracture occurrence.

\section{Sample size}

Based on the results of a similar clinical study, ${ }^{18}$ we predicted a difference in fracture healing time between groups of approximately 1.5 weeks. Assuming a loss to follow-up rate of $20 \%$, each group will need to contain 40 patients to achieve a power of $80 \%$, with an $\alpha$ value of $5 \%$ and $\mathrm{SD}$ of 2.2.

\section{Randomisation procedure}

Randomisation will be requested by the staff member responsible for recruitment. A randomisation tool (www. 
randomization.com) will be used for block randomisation. ${ }^{36}$ For concealment, 80 consecutive numbers will be placed in 80 opaque-sealed envelopes. As each subject is enrolled after verification that they meet the inclusion and exclusion criteria and provision of written informed consent, they will be assigned to a group in accordance with the distribution plan determined by the number contained in the random envelope that they receive. The person who produces and stores the randomly assigned sequence and the investigator who determines the eligibility of the subjects will not be the same person.

\section{Blinding}

This trial is double blinded, which means that both the participants and researchers will be blinded to the intervention. To ensure blinding and compliance, a specific nurse will administer the subcutaneous injection of saline in the placebo group or teriparatide in the treatment group at each visit. The statistician analysing the data will also be blinded to the treatment arms.

\section{Data management}

All study data will be collected and managed in a database created using Epidata. The data will be locked by the main investigator and analyst after confirmation that the data entered into the established database are correct. The locked data file will then be unable to be changed. Any problems detected after the data file has been locked can only be corrected in the statistical analysis program after confirmation of the presence of an error. All studyrelated information will be stored securely at the study site. All physical participant information will be stored in locked file cabinets in areas with limited access. Project principal investigators will have direct access to their own site's data sets.

There is no data monitoring committee for this trial. This is a short-term, early-phase exploratory trial with a relatively small number of subjects. The drug safety (teriparatide) is widely accepted in clinic in current standard dose of treatment. In this trial, we use weekly administration strategy $(40 \mu \mathrm{g} /$ week $)$, significantly reducing the dose from $20 \mu \mathrm{g} /$ day or $40 \mu \mathrm{g} /$ day in other trials. Besides, all the subjects will receive injection in hospital, not at home, which means all the subjects will be under the observation of medical workers. Third, the trial design is direct and straightforward. We only include two groups of patients. There are no complex statistics in this trial. In addition, we still have experienced statisticians in our team to support the trial and data interpretation.

\section{Statistical analysis}

All analyses will be conducted by a statistician who is blinded to the treatment allocations. Primary analyses will be performed using the intention-to-treat population, but a per-protocol analysis will also be conducted. Comparisons between baseline continuous variables will be assessed using the unpaired Student's t-test followed by the Brown-Forsythe test for the equality of group variances; categorical variables will be assessed using Fisher's exact test. The median time taken to achieve radiographic healing and the changes from baseline in the PRWE score and grip strength will be analysed using nonparametric tests. The incidence of fracture non-union will be analysed by the $\mathrm{X}^{2}$ test.

\section{Adverse events and complications}

Safety assessments will include reports of adverse events, serious adverse events, clinical laboratory tests and physical examinations, as well as the follow-up assessments. All the patients will be recruited into a clinical trial insurance provided by Ping An Insurance Company for any extra treatment fee or compensation to those who suffer harm from trial participation.

\section{Modification of the protocol}

Any modifications to the protocol which may impact on the conduct of the study, potential benefit of the patient or may affect patient safety, including changes of study objectives, study design, patient population, sample sizes, study procedures or significant administrative aspects will require a formal amendment to the protocol. Such amendment will be approved by the ethics committee prior to implementation and notified to the health authorities in accordance with local regulations.

\section{ETHICS AND DISSEMINATION}

Ethical approval was obtained from the Peking University Third Hospital Medical Science Research Ethics Committee. There are no interim analyses in this trial. However, the committee will audit trial conduct every other year to track any amendment or serious adverse events and have the right to terminate the trial. The results will be submitted for publication to an international, peer-reviewed journal, regardless of whether the results are positive or negative in relation to the study hypothesis.

Acknowledgements The authors would like to acknowledge Liyuan Tao and Yanyan Shi for their help with the epidemiological and statistical aspects of the study. They also thank Kelly Zammit, from Liwen Bianji, Edanz Editing China (www. liwenbianji.cn/ac), for editing the English text of a draft of this manuscript.

Contributors JZ and CS developed the research question. JZ and CZ contributed to the design of this trial protocol, including the sample size, radiographic evaluation system and functional assessment. JJ and WY made the study flow chart. JZ and MZ helped with the attainment of ethical approval. The manuscript was drafted by $\mathrm{JZ}$ and $\mathrm{CS}$, and was reviewed by all the other authors (including $\mathrm{HL}$ ).

Funding This research is supported by Shenzhen Salubris Pharmaceuticals Co. Competing interests None declared.

Patient consent for publication Not required.

Provenance and peer review Not commissioned; externally peer reviewed.

Supplemental material This content has been supplied by the author(s). It has not been vetted by BMJ Publishing Group Limited (BMJ) and may not have been peer-reviewed. Any opinions or recommendations discussed are solely those of the author(s) and are not endorsed by BMJ. BMJ disclaims all liability and responsibility arising from any reliance placed on the content. Where the content includes any translated material, BMJ does not warrant the accuracy and reliability of the translations (including but not limited to local regulations, clinical guidelines, 
terminology, drug names and drug dosages), and is not responsible for any error and/or omissions arising from translation and adaptation or otherwise.

Open access This is an open access article distributed in accordance with the Creative Commons Attribution Non Commercial (CC BY-NC 4.0) license, which permits others to distribute, remix, adapt, build upon this work non-commercially, and license their derivative works on different terms, provided the original work is properly cited, appropriate credit is given, any changes made indicated, and the use is non-commercial. See: http://creativecommons.org/licenses/by-nc/4.0/.

ORCID iD

Chunli Song http://orcid.org/0000-0002-3690-9457

\section{REFERENCES}

1 Johnell O, Kanis JA. An estimate of the worldwide prevalence and disability associated with osteoporotic fractures. Osteoporos Int 2006;17:1726-33.

2 Heckman JD, Ryaby JP, McCabe J, et al. Acceleration of tibial fracture-healing by non-invasive, low-intensity pulsed ultrasound. J Bone Joint Surg Am 1994;76:26-34.

3 Torstrick FB, Guldberg RE. Local strategies to prevent and treat osteoporosis. Curr Osteoporos Rep 2014;12:33-40.

4 TRUST Investigators writing group, Busse JW, Bhandari M, et al. Re-evaluation of low intensity pulsed ultrasound in treatment of tibial fractures (trust): randomized clinical trial. BMJ 2016;355:i5351.

5 Bishop GB, Einhorn TA. Current and future clinical applications of bone morphogenetic proteins in orthopaedic trauma surgery. Int Orthop 2007;31:721-7.

6 Diefenderfer DL, Osyczka AM, Garino JP, et al. Regulation of BMPinduced transcription in cultured human bone marrow stromal cells. $J$ Bone Joint Surg Am 2003;85-A Suppl 3:19-28.

7 Carragee EJ, Hurwitz EL, Weiner BK. A critical review of recombinant human bone morphogenetic protein-2 trials in spinal surgery: emerging safety concerns and lessons learned. Spine $J$ 2011;11:471-91.

8 Carragee EJ, Chu G, Rohatgi R, et al. Cancer risk after use of recombinant bone morphogenetic protein-2 for spinal arthrodesis. $J$ Bone Joint Surg Am 2013;95:1537-45.

9 Aro HT, Govender S, Patel AD, et al. Recombinant human bone morphogenetic protein-2: a randomized trial in open tibial fractures treated with reamed nail fixation. J Bone Joint Surg Am 2011;93:801-8.

10 Krell ES, DiGiovanni CW. The efficacy of platelet-derived growth factor as a Bone-Stimulating agent. Foot Ankle Clin 2016;21:763-70.

11 Aspenberg P. Special review: accelerating fracture repair in humans: a reading of old experiments and recent clinical trials. Bonekey Rep 2013;2:244.

12 Dede AD, Makras P, Anastasilakis AD. Investigational anabolic agents for the treatment of osteoporosis: an update on recent developments. Expert Opin Investig Drugs 2017;26:1137-44.

13 Andreassen TT, Ejersted C, Oxlund H. Intermittent parathyroid hormone (1-34) treatment increases callus formation and mechanical strength of healing rat fractures. J Bone Miner Res 1999;14:960-8.

14 Holzer G, Majeska RJ, Lundy MW, et al. Parathyroid hormone enhances fracture healing. Clin Orthop Relat Res 1999;366:258-63.

15 Nakajima A, Shimoji N, Shiomi K, et al. Mechanisms for the enhancement of fracture healing in rats treated with intermittent low-dose human parathyroid hormone (1-34). J Bone Miner Res 2002;17:2038-47.

16 Nakazawa T, Nakajima A, Shiomi K, et al. Effects of low-dose, intermittent treatment with recombinant human parathyroid hormone (1-34) on chondrogenesis in a model of experimental fracture healing. Bone 2005;37:711-9.
17 Skripitz R, Andreassen TT, Aspenberg P. Parathyroid hormone (1-34) increases the density of rat cancellous bone in a bone chamber. A dose-response study. J Bone Joint Surg Br 2000;82:138-41.

18 Aspenberg P, Genant HK, Johansson T, et al. Teriparatide for acceleration of fracture repair in humans: a prospective, randomized, double-blind study of 102 postmenopausal women with distal radial fractures. J Bone Miner Res 2010;25:404-14.

19 Aspenberg P, Johansson T. Teriparatide improves early callus formation in distal radial fractures. Acta Orthop 2010;81:234-6.

20 Peichl P, Holzer LA, Maier R, et al. Parathyroid hormone 1-84 accelerates fracture-healing in pubic bones of elderly osteoporotic women. J Bone Joint Surg Am 2011;93:1583-7.

21 Almirol EA, Chi LY, Khurana B, et al. Short-term effects of teriparatide versus placebo on bone biomarkers, structure, and fracture healing in women with lower-extremity stress fractures: a pilot study. J Clin Transl Endocrinol 2016;5:7-14.

22 Yoo J-I, Ha Y-C, Ryu H-J, et al. Teriparatide treatment in elderly patients with sacral insufficiency fracture. J Clin Endocrinol Metab 2017;102:560-5.

23 Shiraki M, Sugimoto T, Nakamura T. Effects of a single injection of teriparatide on bone turnover markers in postmenopausal women. Osteoporos Int 2013;24:219-26.

24 Nakamura T, Sugimoto T, Nakano T, et al. Randomized teriparatide [human parathyroid hormone (PTH) 1-34] once-weekly efficacy research (TOWER) trial for examining the reduction in new vertebral fractures in subjects with primary osteoporosis and high fracture risk. J Clin Endocrinol Metab 2012;97:3097-106.

25 Sugimoto T, Shiraki M, Nakano T, et al. Vertebral fracture risk after once-weekly teriparatide injections: follow-up study of teriparatide once-weekly efficacy research (tower) trial. Curr Med Res Opin 2013;29:195-203.

26 Fujita T, Fukunaga M, Itabashi A, et al. Once-weekly injection of lowdose teriparatide $(28.2 \mu \mathrm{g})$ reduced the risk of vertebral fracture in patients with primary osteoporosis. Calcif Tissue Int 2014;94:170-5.

27 Ebata S, Takahashi J, Hasegawa T, et al. Role of Weekly teriparatide administration in osseous Union enhancement within six months after posterior or Transforaminal lumbar interbody fusion for Osteoporosis-Associated lumbar degenerative disorders: a multicenter, prospective randomized study. J Bone Joint Surg Am 2017;99:365-72.

28 Neer RM, Arnaud CD, Zanchetta JR, et al. Effect of parathyroid hormone (1-34) on fractures and bone mineral density in postmenopausal women with osteoporosis. N Engl J Med 2001;344:1434-41.

29 Chen P, Satterwhite JH, Licata AA, et al. Early changes in biochemical markers of bone formation predict BMD response to teriparatide in postmenopausal women with osteoporosis. J Bone Miner Res 2005;20:962-70.

30 Zhang W, Zhu J, Ma T, et al. Comparison of the effects of onceweekly and once-daily rhPTH (1-34) injections on promoting fracture healing in rodents. J Orthop Res 2018;36:1145-1152.

31 Johansson T. Pth 1-34 (teriparatide) may not improve healing in proximal humerus fractures. A randomized, controlled study of 40 patients. Acta Orthop 2016;87:79-82.

32 Ota M, Takahata M, Shimizu T, et al. Optimal administration frequency and dose of teriparatide for acceleration of biomechanical healing of long-bone fracture in a mouse model. $J$ Bone Miner Metab 2019;37:256-63.

33 Kay S, McMahon M, Stiller K. An advice and exercise program has some benefits over natural recovery after distal radius fracture: a randomised trial. Aust J Physiother 2008;54:253-9.

34 Litrenta J, Tornetta P, Mehta S, et al. Determination of radiographic healing: an assessment of consistency using rust and modified rust in Metadiaphyseal fractures. J Orthop Trauma 2015;29:516-20.

35 MacDermid JC, Turgeon T, Richards RS, et al. Patient rating of wrist pain and disability: a reliable and valid measurement tool. $J$ Orthop Trauma 1998;12:577-86.

$36 \mathrm{Kim}$ J, Shin W. How to do random allocation (randomization). Clin Orthop Surg 2014;6:103-9. 